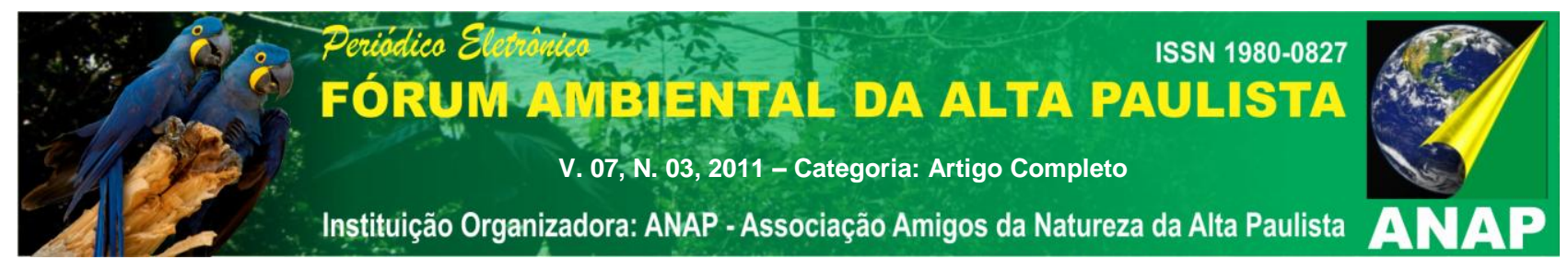

\title{
O PARQUE KRUGER DA AFRICA DO SUL E SUA AVIFAUNA
}

\author{
Alvarez Pereira Arjona ${ }^{1}$
}

Sueli Yoshinaga Pereira ${ }^{2}$

Paulo Ricardo Brum Pereira ${ }^{3}$

RESUMO: O presente estudo objetivou a identificação da avifauna dentro do Parque Nacional Kruger e seu entorno, na África do Sul. A África do Sul é um país com uma rica Avifauna, tendo o registro de 958 espécies. O Parque Kruger é um dos mais importantes parques da vida selvagem do mundo. Já foram registradas mais de 500 espécies da Avifauna no seu interior. Este estudo foi desenvolvido nos anos de 2008, 2009, 2010 e 2011 e os procedimentos metodológicos foram o registro com fotos e identificação com bibliografia especializada. Foram feitas no mínimo 15 viagens, em um total mínimo de 200 horas de observações em campo. Foram fotografadas e identificadas 107 espécies da avifauna, e apresentadas algumas fotos.

Palavras - chave: Avifauna, Kruger Park, África do Sul.

\footnotetext{
${ }^{1}$ Suboficial. Força Aérea Brasileira. alvarezparjona@gmail.com.

${ }^{2}$ Geóloga. Instituto de Geociências/ Unicamp. Profa. Dra. sueliyos@ige.unicamp.br

${ }^{3}$ Geógrafo. Instituto Florestal/ SMA. Pesquisador Científico, Dr. paulobrumpereira@ig.com.br
} 


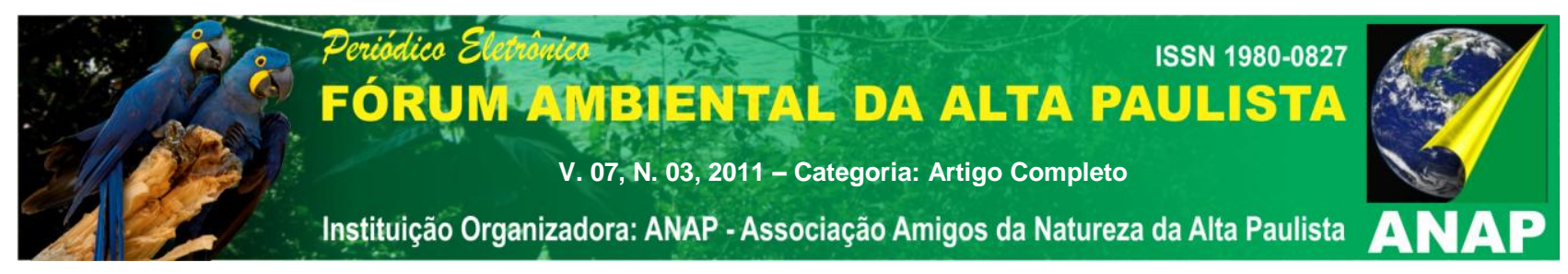

\section{INTRODUÇÃO}

O presente estudo foi realizado no Parque Kruger (PK) e seu entorno, na África do Sul. E teve como principal objetivo a observar, fotografar e identificar a avifauna desse parque sul africano.

O Parque Kruger, foi fundado em 1898, e seu nome é uma homenagem ao presidente Paul Kruger, que declarou a área como parque nacional. Possuí uma área de 19 mil km², é maior que países como Suiça, Israel e País de Gales. Está localizado no extremo nordeste do país, nas províncias de Limpopo e Mpumalanga. Estende-se por mais de $380 \mathrm{~km}$ do norte ao sul (ao norte começa no Limpopo River e termina ao sul no Crocodile River), com uma largura média aproximada de $60 \mathrm{~km}$. É limítrofe e interligado formando um corredor de biodiversidade com os Parques do Limpopo (Moçambique) e Gonarezhou (Zimbabwe). Os três formam o grande Parque Transfronteiriço do Limpopo, criado em 2002. (www.africadosul.org.br; www.krugerpark.co.za/great-limpopo-transfrontierpark-so) (Figura 01). 

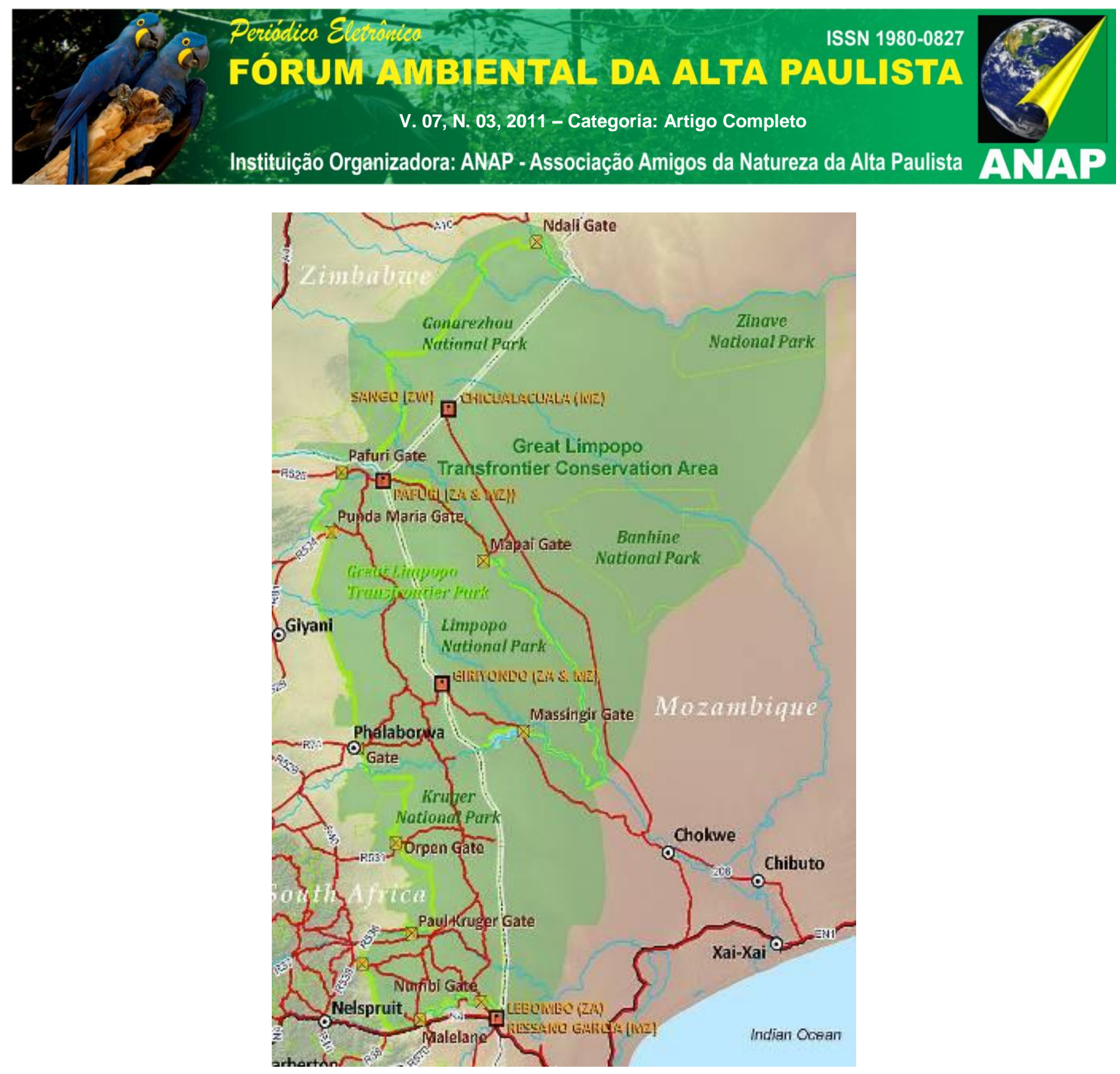

Figura 01 - Parque Transfronteiriço do Limpopo (Escala aproximada 1:450.000).

O PK possui dezesseis ecossistemas diferenciados em termos de geologia, geomorfologia, clima e vegetação. Abriga mais de 500 espécies de aves, 147 de mamíferos (em torno de 2000 leões, 1000 leopardos, 1800 rinocerontes, 8000 elefantes e 22000 búfalos que são os cinco grandes os "big fives") e 112 de répteis e 49 de peixes (http://en.wikipedia.org/wiki/Kruger_National_Park). O clima do PK é tropical seco ao norte tendendo ao subtropical menos seco para sul. O período de setembro a maio é úmido e quente, com temperaturas que podem superar os $38^{\circ} \mathrm{C}$. A estação seca e fria vai de maio a agosto. A média de precipitação anual varia entre 400 (norte) a $750 \mathrm{~mm}$ (sul). (VENTER \& GERTENBACH, 2008; www.africadosul.org.br; www.krugerpark.co.za/geological-krugermap.html). Segundo Venter \& Gertenbach (2008), a vegetação do PK é composta de aproximadamente 1968 espécies e a diversidade da vegetação não se manifesta em um 


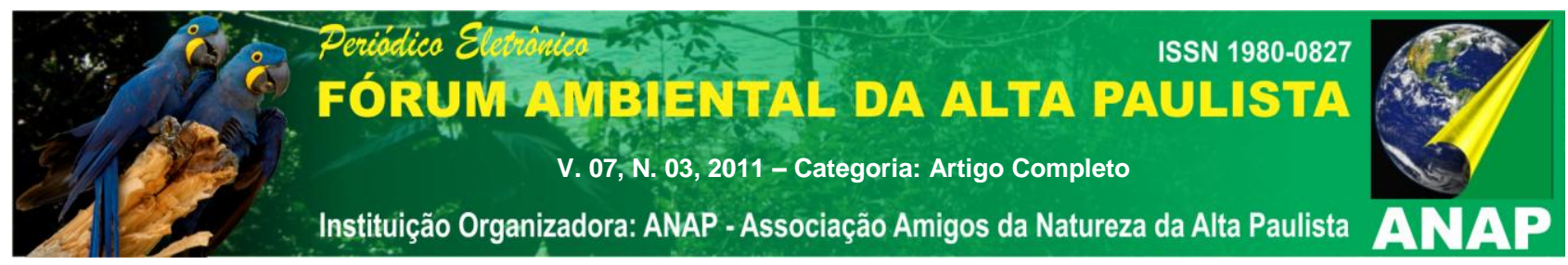

grande número de plantas, porém se manifesta em uma grande variedade de feições estruturais, desde densas florestas abertas bem como feições de vegetação arbustiva espaçada com gramíneas. Algo parecido com a vegetação brasileira do Cerradão e Cerrado Tipico.

\section{METODOLOGIA}

Basicamente os procedimentos técnicos e metodológicos foram:

2.1. A escolha do Parque Kruger e seu entorno (menos de $5 \mathrm{~km}$ das cercas elétricas do Parque) como a Unidade de Análise para a identificação da Avifauna (somente na África do Sul);

2.2. Assumiu-se que a análise deveria ser feita em todas as estações do ano, para se registrar a avifauna em períodos secos e úmidos;

2.3. Adotou-se o procedimento do registro da avifauna, somente no período diurno, de forma visual, e também com binóculos e com câmeras fotográficas e registro em caderneta de campo. Quando a ave era avistada, imediatamente se fotografava, e posteriormente se tentava melhores ângulos, boa luminosidade e proximidade;

2.4. Imediatamente (quando possível) após o registro e com apoio da bibliografia, sobre a avifauna na área específica, procedia-se a identificação;

2.5. No entorno do Parque os deslocamentos para os registros foram feitos de carro e a pé, e dentro do Parque somente de carro (por motivo da lei e da segurança). Em áreas específicas do Parque os deslocamentos podiam ser feitos a pé, pois era seguro. O carro foi um modelo Ford K, 2 portas;

2.6. No período seco, dava-se mais atenção às áreas com disponibilidade de água, quando ocorria a concentração dos animais. A velocidade do carro nunca era superior a $40 \mathrm{~km}$ por hora; 


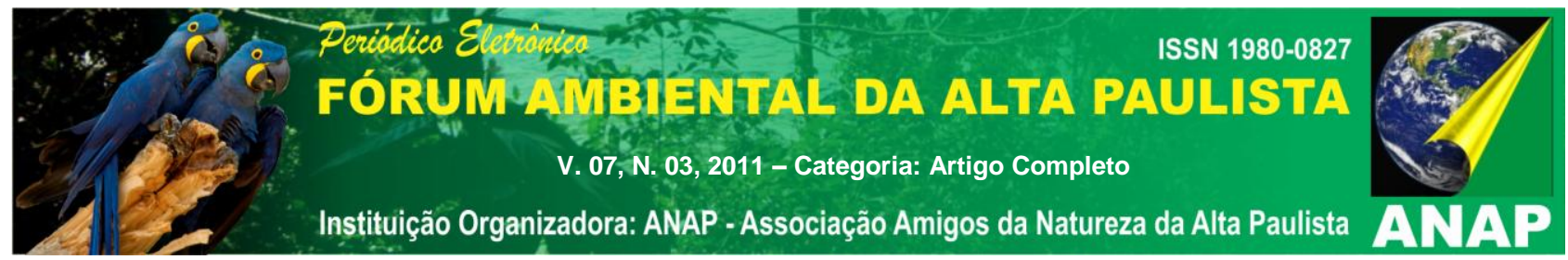

2.7. Foram realizadas mais de 15 viagens, perfazendo um total mínimo de 200 horas de observações, em todas as estações dos anos de 2008, 2009, 2010 e 2011. Em 2010 e 2011 todos os autores presentes. O primeiro autor presente em todos os anos;

2.8. Em média foram fotografadas 220 aves por dia, perfazendo um total aproximado de 3300 fotos nas 15 viagens feitas (esclarecendo que as fotos poderiam ser repetidas várias vezes da mesma ave em cada viagem, técnica para facilitar a identificação posterior).

\section{ANÁLISE E DISCUSSÕES}

A riqueza da avifauna do PK é imensa, e a titulo comparativo com 04 biomas brasileiros e 02 estudos específicos realizados recentemente em São Paulo e Rio Grande do Sul apresentamos a Tabela 1.

Tabela 1. Comparativo entre o Parque Kruger, África do Sul (1) e alguns grandes biomas brasileiros (4, 5, 6 e 7) e dois estudos recentes realizados no estado de São Paulo (2) e Rio Grande do sul (3)

\begin{tabular}{lcc}
\hline \multicolumn{1}{c}{ Áreas Comparativas } & Área $\left(\mathrm{Km}^{2}\right)$ & № espécies da Avifauna \\
\hline 1. Parque Kruger & 21.000 & 507 \\
2. RM Campinas SP & 3.647 & 202 \\
3. BR Toropi RS & 3.800 & 245 \\
4. Bioma Pantanal & 137.000 & 656 \\
5. Bioma Pampa & 170.000 & 476 \\
6. Bioma Mata Atlântica & $1.110,182$ & 1.020 \\
7. Bioma Cerrado & 2.000 .000 & 837
\end{tabular}

Fontes: 1 www.africadosul.org.br; 2 Pereira et al. (2009); 3 Pereira et al. (2009); 4, 5, 6 e 7 www.ibama.gov.br ou www.eptv.globo.com/terradagente.

A avifauna identificada no Parque Kruger, está apresentada na Tabela 2, abaixo. Todos os nomes populares foram traduzidos para o português, exceto o Buteo vulpinus e B.trizonatus (Familia Accipitridae) e Onychognatus morio (Família Sturnidae). 


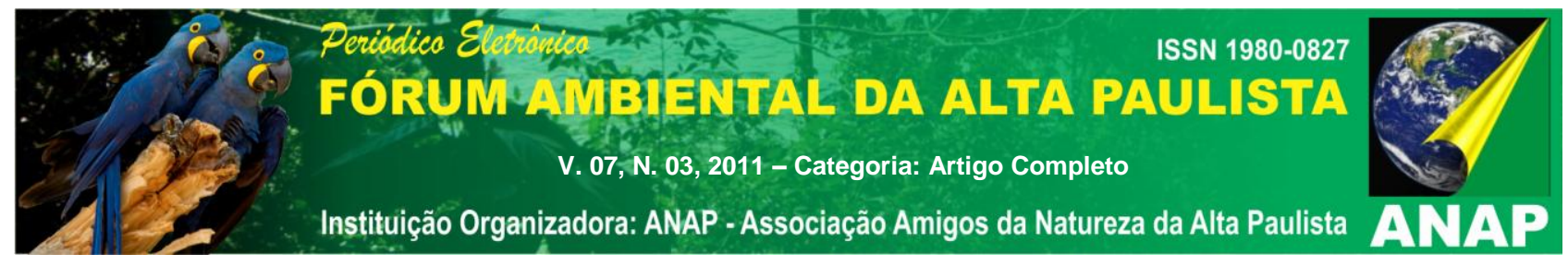

Tabela 2 - Famílias e espécies classificadas na região de estudo

\begin{tabular}{|c|c|c|}
\hline Família & Nome científico & Nome popular traduzido \\
\hline \multirow[t]{13}{*}{ Accipitridae } & Lophaetus occiptalis & Águia-de-poupa \\
\hline & Aquila rapax & Águia-rapace \\
\hline & Circaetus pectoralis & $\begin{array}{l}\text { Águia-cobreira-do-peito- } \\
\text { preto }\end{array}$ \\
\hline & Aquila wahlbergi & Águia-de-wahlberg \\
\hline & Aquila pennatus & Águia-calçada \\
\hline & Buteo vulpinus & \\
\hline & Haliaeetus vocifer & Águia-pescadora \\
\hline & Melierax metabates & Açor-cantor \\
\hline & Buteo trizonatus & \\
\hline & Polyboroides typus & Secretário-pequeno \\
\hline & Necrosyrtes monachus & Abutre-de-capuz \\
\hline & Gyps africanus & Grifo-do-cabo \\
\hline & Gyps coprotheres & Abutre-do-cabo \\
\hline \multirow[t]{2}{*}{ Alcedinidae } & Alcedo cristata & Pica-peixe-de-poupa \\
\hline & Ceryle rudis & Martim-pescador \\
\hline \multirow[t]{6}{*}{ Anatidae } & Alopochen aegyptiacus & Ganso-do-egito \\
\hline & Anas capensis & Marreco-do-cabo \\
\hline & Dendrocygna viduata & Irerê, Marrecão (1) \\
\hline & Anas hottentota & Marreco-hotentote \\
\hline & Anas sparsa & Pato-preto-africano \\
\hline & Anas undulata & Pato-do-bico-amarelo \\
\hline Anhingidae & Anhinga rufa & Mergulhão-serpente \\
\hline \multirow[t]{3}{*}{ Ardeidae } & Ardea cinerea & Garça-real (1) \\
\hline & Bubulcus ibis & Garça-vaqueira (1) \\
\hline & Butorides striata & Socózinho (1) \\
\hline
\end{tabular}




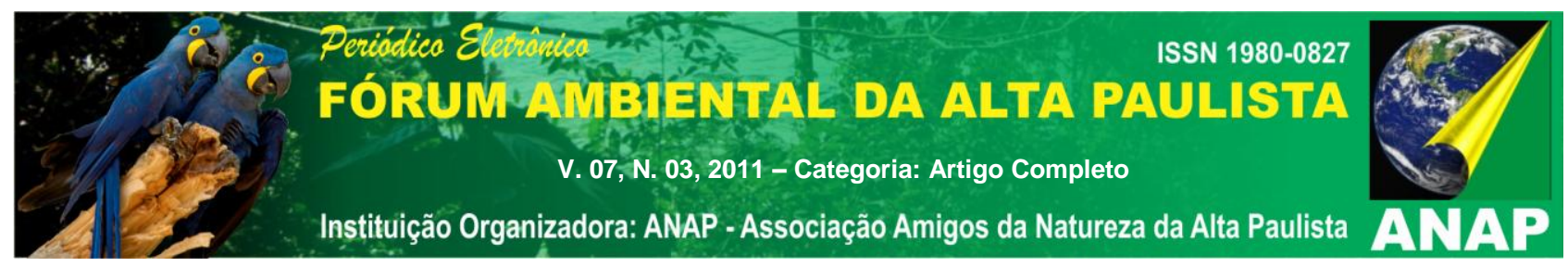

\begin{tabular}{lll}
\hline \multicolumn{1}{c}{ Família } & \multicolumn{1}{c}{ Nome científico } & Nome popular traduzido \\
\hline Egretta Alba & Garça-branca (1) \\
Egretta intermédia & Garça-branca-intermédia \\
Ixobrychus minutus & Garça-pequena \\
Nycticorax nycticorax & Savacu (1) \\
Bucerotidae & $\begin{array}{l}\text { Bucorvus leadbeateri } \\
\text { Tockus leucomella }\end{array}$ & Bico-de-corno \\
& Calau-de-bico-vermelho \\
Bockus alboterminatus & Calau-coroado \\
& Calau-trombeteiro
\end{tabular}

\begin{tabular}{|c|c|c|}
\hline \multirow[t]{2}{*}{ Burhinidae } & Burhinus capensis & Alcaravão-do-cabo \\
\hline & Burhinus vermiculatus & Alcaravão-d'água \\
\hline \multirow[t]{7}{*}{ Charadriidae } & Vanellus senegallus & Abibe-do-senegal \\
\hline & Vanellus armatus & Abibe-preto-ebranco \\
\hline & Vanellus coronatus & Abibe-coroado \\
\hline & Vanellus melanopterus & Abibe \\
\hline & Vanellus Iugubris & Abibe \\
\hline & Charadrius marginatus & Borrelho-de-testa-branca \\
\hline & Charadrius tricollaris & Borrelho-de-tripla-coleira \\
\hline
\end{tabular}

\begin{tabular}{lll} 
Ciconiidae & $\begin{array}{l}\text { Ciconia episcopus } \\
\text { Ciconia ciconia } \\
\text { Mycteria ibis }\end{array}$ & $\begin{array}{l}\text { Cegonha-episcopal } \\
\text { Cegonha-branca } \\
\text { Cegonha-de-bico-amarelo }\end{array}$ \\
\hline Ciconiidae & Leptoptilos crumeníferus & Marabu \\
Coliidae & Oena capensis & Rabo-de-junco-estriado \\
Columbidae & $\begin{array}{l}\text { Streptopelia decipiens } \\
\text { Streptopelia Semitorquata }\end{array}$ & $\begin{array}{l}\text { Rola-gemedora } \\
\text { Rola-de-olhos-vermelhos }\end{array}$ \\
\end{tabular}




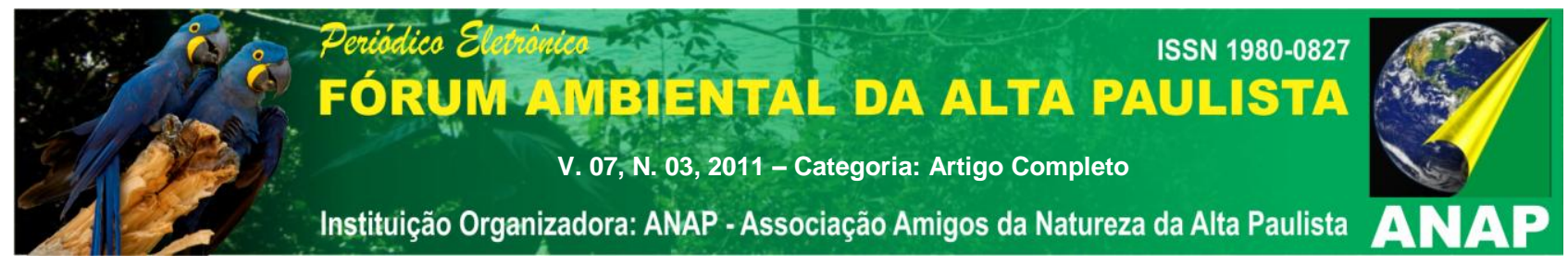

\begin{tabular}{|c|c|c|}
\hline Família & Nome científico & Nome popular traduzido \\
\hline & Streptopelia Capicola & Rola-do-cabo \\
\hline & Streptopelia senegalensis & Rola-do-senegal \\
\hline & Sreptopelia guinea & Pomba-da-guiné \\
\hline & Streptopelia arquatrix & Pomba-de-olho-amarelo \\
\hline \multirow[t]{3}{*}{ Coraciidae } & Coracias caudatas & Rolieiro-de-peito-lilás \\
\hline & Coracias spatulatus & Rolieiro-cauda-de-raquete \\
\hline & Coracias garrulus & Rolieiro \\
\hline \multirow[t]{3}{*}{ Corvidae } & Corvus albicollis & Corvo-da-montanha \\
\hline & Corvus albus & Gralha-seminarista \\
\hline & Corvus capensis & Gralha-do-cabo \\
\hline \multirow[t]{2}{*}{ Emberizidae } & Emberiza capensis & Escrevedeira-do-cabo \\
\hline & Emberiza tahapisi & $\begin{array}{l}\text { Escrevedeira-de-peito- } \\
\text { canelado }\end{array}$ \\
\hline \multirow[t]{3}{*}{ Falconidae } & Falco amurensis & Falcão \\
\hline & Falco peregrinus & Falcão-peregrino (1) \\
\hline & Falco subbuteo & Ógea \\
\hline \multirow[t]{2}{*}{ Glareolidae } & Rhinoptilus chalcopterus & Corredor-de-pata-vermelha \\
\hline & Cursorius temminckii & Corredor-de-temminck \\
\hline \multirow[t]{2}{*}{ Gruidae } & Balearica regulorum & Grou \\
\hline & Bugeranus carunculatus & Grou-carunculado \\
\hline Jacanidae & Actophilornis africanus & Jaçanã-africana \\
\hline Laniidae & Lanius souzae & Picanço-de-souza \\
\hline Meropidae & Merops apiaster & Abelharuco \\
\hline
\end{tabular}




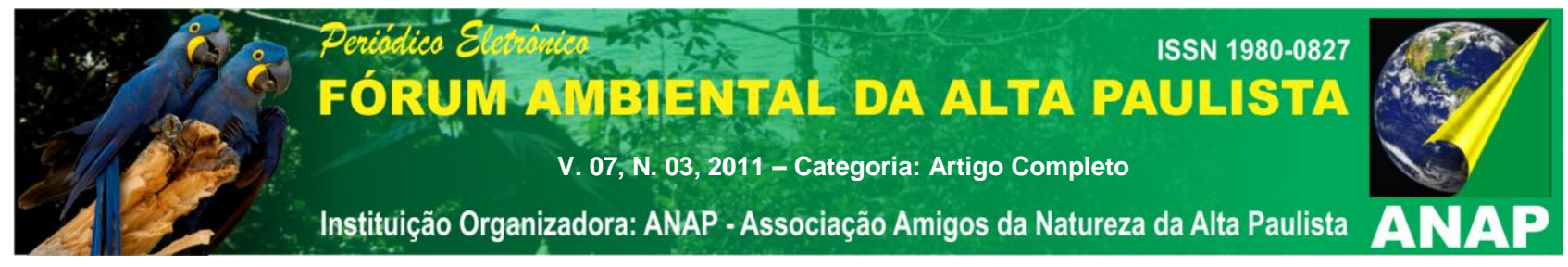

\begin{tabular}{|c|c|c|}
\hline Família & Nome científico & Nome popular traduzido \\
\hline & Merops bullockoides & $\begin{array}{l}\text { Abelharuco-de-testa- } \\
\text { branca }\end{array}$ \\
\hline
\end{tabular}

\begin{tabular}{|c|c|c|}
\hline Motacilidae & Motacilla aguimp & Alveola-africana \\
\hline Musophagidae & Tauraco livingstoni & Turaco-de-livingstone \\
\hline Numididae & Numida meleagris & Galinha-d`angola \\
\hline \multirow[t]{4}{*}{ Otididae } & Lissotis melanogaster & Sisão-de-barriga-preta \\
\hline & Eupodotis caerulescens & Abetarda-azul \\
\hline & Eupodotis barrow & $\begin{array}{l}\text { Abetarda-de-barriga- } \\
\text { branca }\end{array}$ \\
\hline & Lophotis ruficrista & Abetarda-de-poupa \\
\hline Phalacrocoracidae & Phalacrocorax africanus & Mergulão-serpente \\
\hline \multirow[t]{7}{*}{ Phasianidae } & Pternistis swainsoni & Francolim \\
\hline & Dendroperdix sephaena & Francolim-de-poupa \\
\hline & Scleroptila africanus & Francolim-d'asa-cinzenta \\
\hline & Pternist afer & $\begin{array}{l}\text { Francolim-de-pescoço- } \\
\text { vermelho }\end{array}$ \\
\hline & Scleroptila shelleyi & Francolim-de-shelley \\
\hline & Peliperdix coqui & Francolim-das-pedras \\
\hline & Pternistis natalensis & Francolim-do-natal \\
\hline \multirow[t]{3}{*}{ Ploceidae } & Ploceus intermedius & $\begin{array}{l}\text { Tecelão- pequeno-de- } \\
\text { máscara }\end{array}$ \\
\hline & Euplectes ardens & Viúva-de-colar-vermelho \\
\hline & Ploceus velatus & Tecelão-de-máscara \\
\hline
\end{tabular}




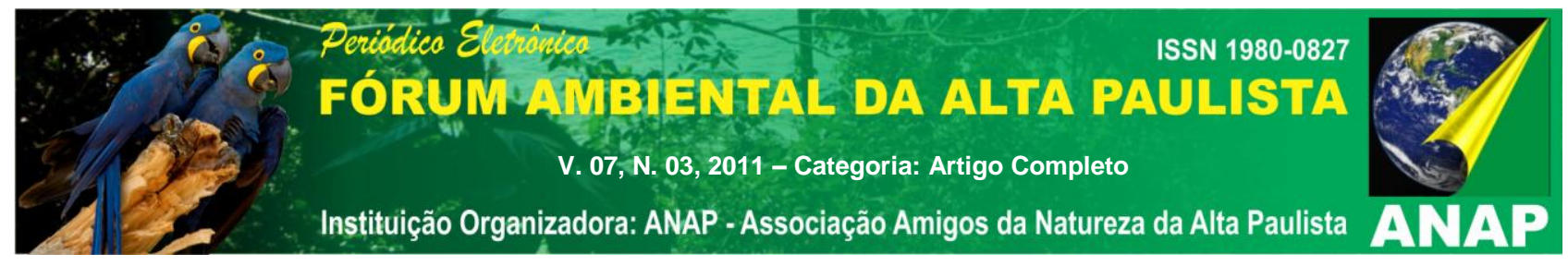

\begin{tabular}{|c|c|c|}
\hline Família & Nome científico & Nome popular traduzido \\
\hline \multirow[t]{6}{*}{ Rallidae } & Gallinula angulata & Frango-d'água \\
\hline & Rallus caerulescens & Frango-d'água \\
\hline & Amaurornis flavirostris & Frango-d’àgua-preto \\
\hline & Porphyrio & Caimão-comum \\
\hline & madagascariensis & \\
\hline & Gallinula chloropus & Frango- d’àgua (1) \\
\hline Sagittariidae & Sagittarius serpentarius & Ave-secretária \\
\hline Struthionidae & Struthio camelus & Avestruz \\
\hline \multirow[t]{2}{*}{ Sturnidae } & Onychognatus morio & \\
\hline & Buphagus erythrorhynchus & Pica-boi \\
\hline \multirow[t]{4}{*}{ Threskiornithidae } & Bostrychia hagedash & Íbis \\
\hline & Geronticus calvus & Ibis-careca \\
\hline & Plegadis falcinellus & Ibis-preta \\
\hline & Threskiornis aethiopicus & Ibis-sagrada \\
\hline Turnicidae & Turnix sylvatica & Codorna \\
\hline
\end{tabular}

No período de estudo foram identificadas 34 famílias da avifauna do Parque Kruger, com um total de 107 espécies, perfazendo um total de aproximadamente 20\% de toda a avifauna do parque. Número considerado significativo devido às circunstâncias em que o estudo foi desenvolvido e os objetivos propostos. No entorno do Parque era possível a livre circulação para o estudo, mas dentro do Parque os trajetos eram percorridos de carro, e somente em pontos específicos era possível sair do veiculo para o trabalho. Acresce-se a isso o fato de que o modelo do veículo (Ford K, 2 portas), não era 


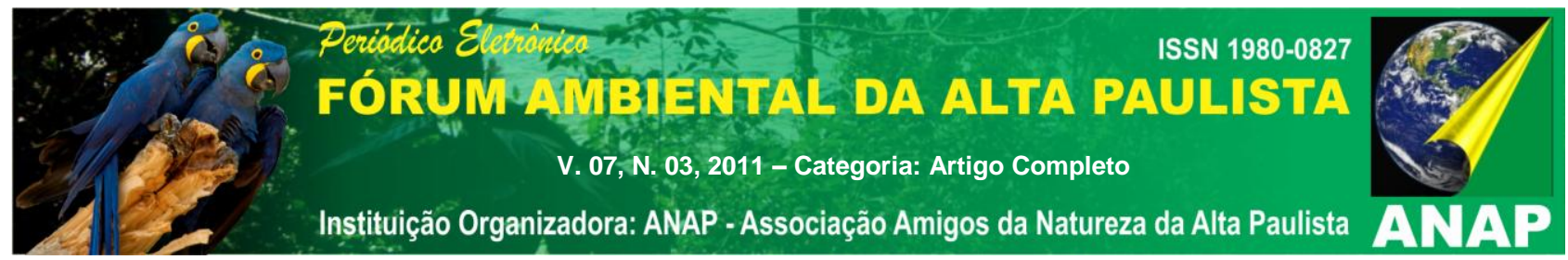

o mais adequado para um estudo da avifauna, mas foi feito o que era possível. O ideal seria um veículo tracionado, sem cobertura, para se chegar a pontos mais isolados.

Foi descartado um número muito grande de fotos e registros em caderneta de campo, principalmente da avifauna das famílias Accipitridae e Falconidae (Gaviões, Falcões e Águias), Apodidae (Andorinhas) e Trochilidae (Beija-flores), esse procedimento foi assumido como adequado quando surgia algum tipo de dúvida sobre a identificação. $\mathrm{O}$ mesmo ocorrendo com toda a avifauna com hábitos noturnos.

Ao menos 08 espécies da avifauna encontradas no Parque Kruger são encontradas também no Estado de São Paulo, são elas:

O Frango-d’água (Gallinula chloropus), o Paturi ou Marrecão (Dendrocygna viduata), a Garça-real (Ardea cinerea), a Garça-Vaqueira (Bubulcus ibis), o Socózinho (Butorides striata), a Garça-branca (Egretta Alba), o Savacu (Nycticorax nycticorax) e o Falcão-peregrino (Falco peregrinus).

Apresentamos, ao final do estudo, um total de 16 figuras (Figura 2 até 17), com o objetivo de ilustrar o estudo. As fotos foram feitas pelos autores.

Com o objetivo de trazermos a nossa experiência e a nossa contribuição ao conhecimento da avifauna de um dos mais importantes Parques da Vida Selvagem de todo o mundo, é que apresentamos o presente estudo para a comunidade científica brasileira e para os amantes da observação da avifauna.

\section{REFERÊNCIAS}

Pereira, P.R.B.; Yoshinaga-Pereira, S.; De Freitas, J.A.; Toledo Filho, D. V. Análise Ambiental, Aspectos Abióticos, Vegetação e Avifauna na Região Metropolitana de Campinas: Subsídios à Elaboração dos Planos de Manejonda Estação Ecológica de Valinhos (EE Valinhos) e do Parque Estadual da Assessoria da Reforma Agrária (PE ARA), Valinhos, SP. V Fórum Ambiental da Alta Paulista, ISSN 1980-0827. p.299 - 314. 2009. 


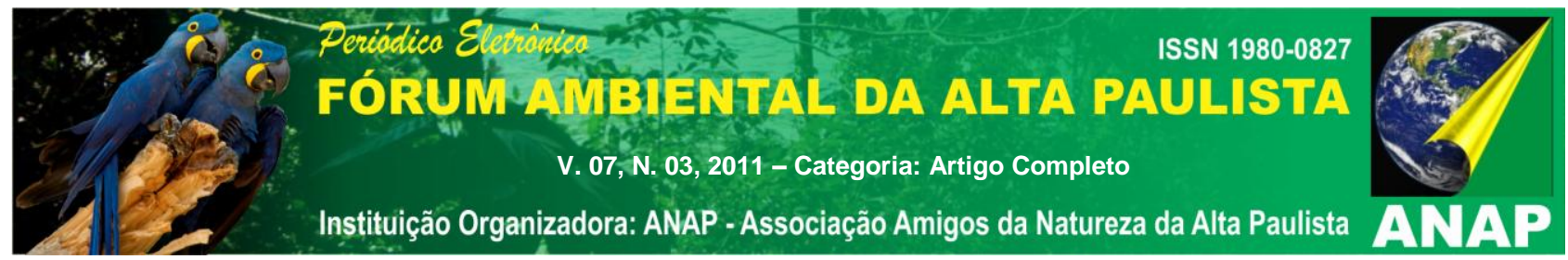

Pereira, P.H.V.; Pereira, P.R.B.; Yoshinaga-Pereira, S.; Distribuição Geográfica da Avifauna na Bacia Hidrográfica do Rio Toropi - RS. V Fórum Ambiental da Alta Paulista, ISSN 1980-0827. p.149 -166. 2009.

Sinclair, I.; Ryan, P. Complete Photographic Field Guide Birds of Southern Africa. Struik Publishers. South Africa, Cape Town. 432p. 2009.

Sinclair, I.; Hockey, P. The Larger Illustrated Guide to Birds of Southern Africa. Struik Publishers. South Africa, Cape Town. 2005.

Sinclair, I.; Hockey, P. ; Tarboton, W. Birds of Southern Africa. Struik Publishers. South Africa, Cape Town. 426p. 1993.

Venter, F., Gertenbach, W.. A. Cursory Review of the Climate and Vegetation of the Kruger National Park. Koedoe - African Protected Area Conservation and Science, North America, 29, nov. 2008. Available at: www.koedoe.co.za/index.php/koedoe/article/view/526>. Date accessed: 30 Jul. 2011. www.krugerpark.co.za/great-limpopo-transfrontier-park-so. Acesso: Maio de 2011 www.africadosul.org.br. Acesso: Maio de 2011 www.krugerpark.co.za/geological-kruger-map.html Acesso: Junho de 2011 www.en.wikipedia.org/wiki/Kruger_National_Park. Acesso: Maio 2011 www.avibase.bsc-eoc.org/avibase.jsp?pg=home\&lang=PT Acesso: Junho e Julho 2011. www.dazacarias.blogspot.com Acesso: Julho 2011 

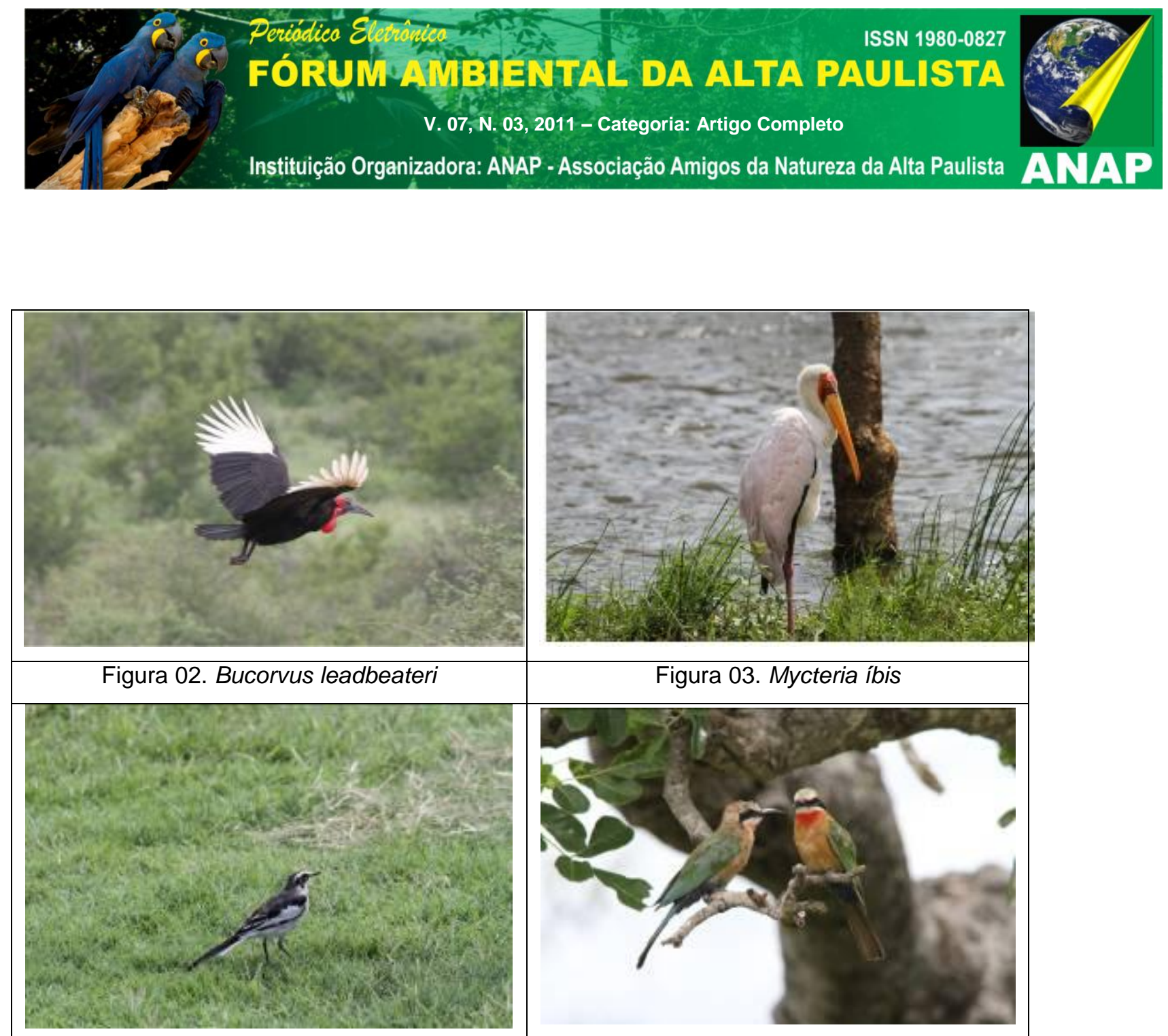

Figura 03. Mycteria íbis

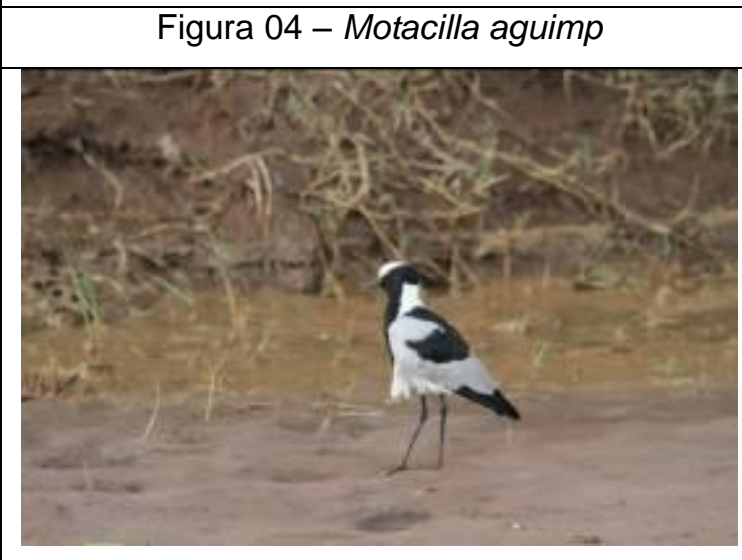

Figura 06 - Vanellus armatus
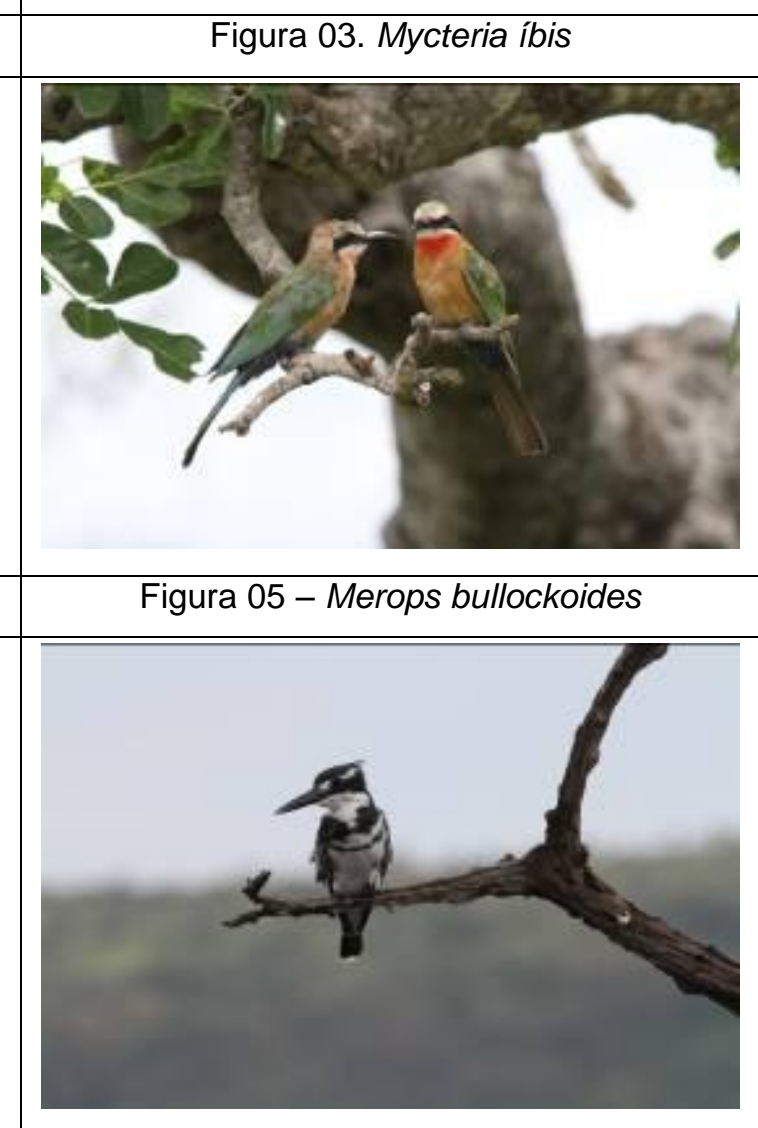

Figura 07 - Ceryle rudis 

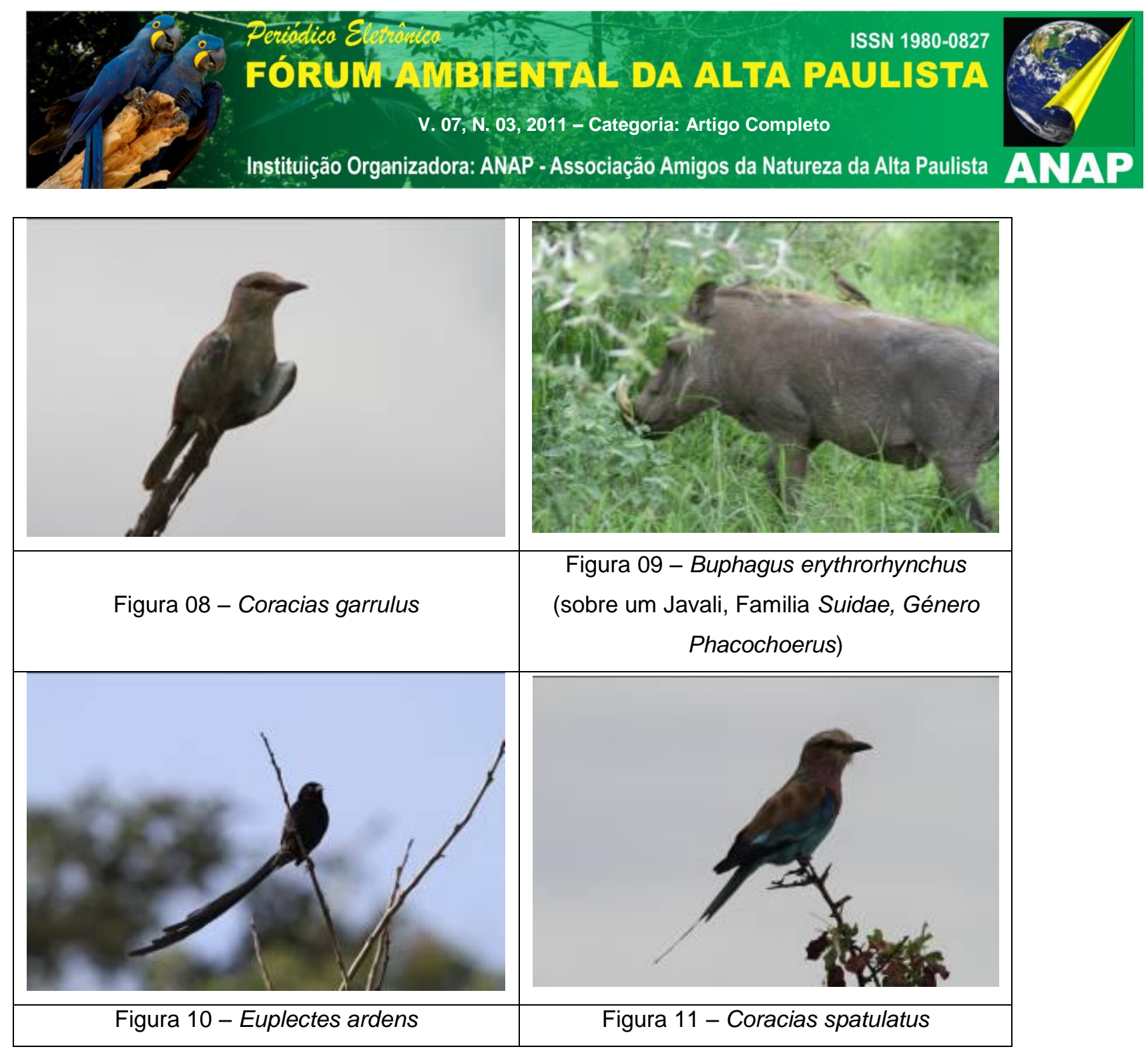

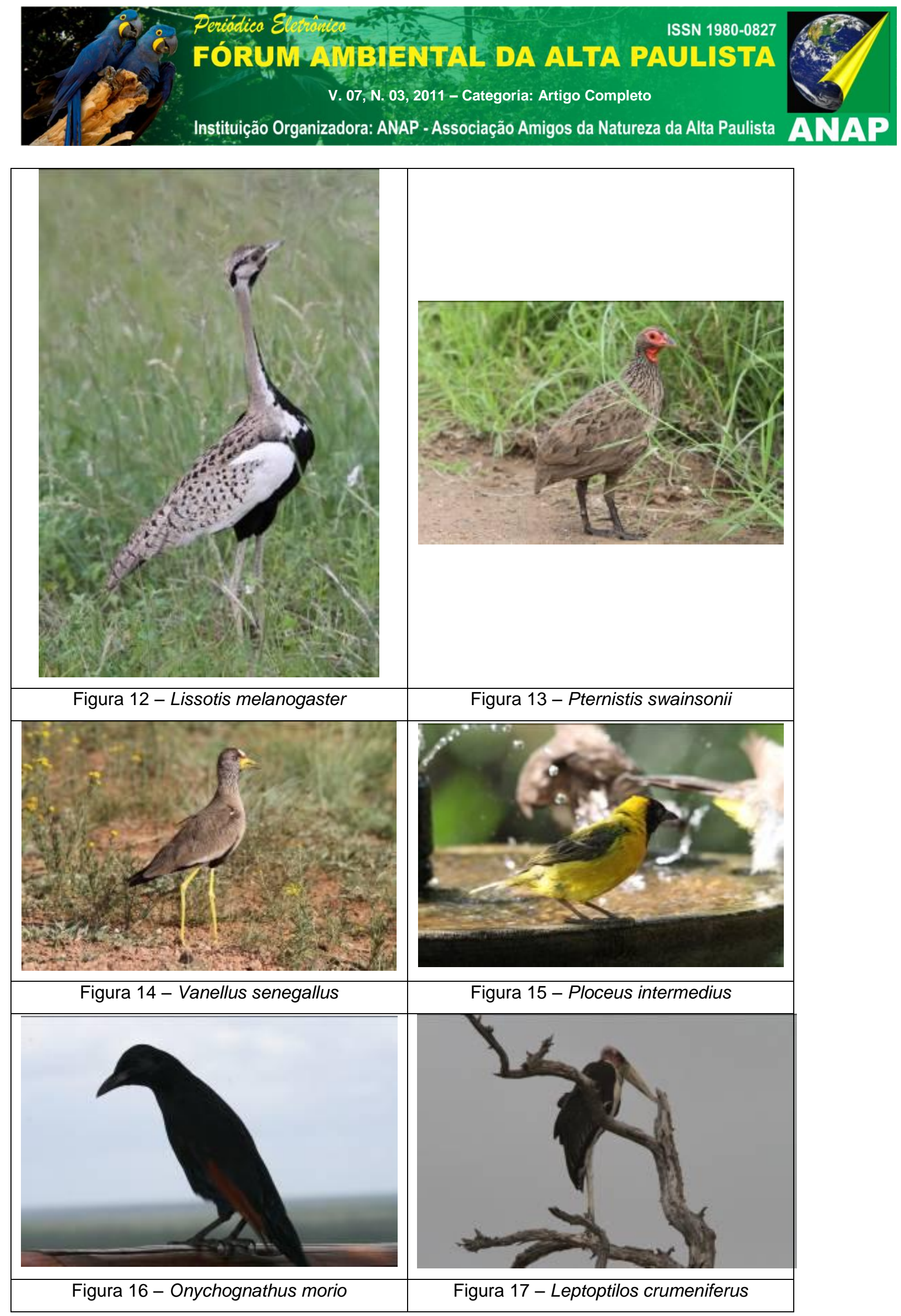


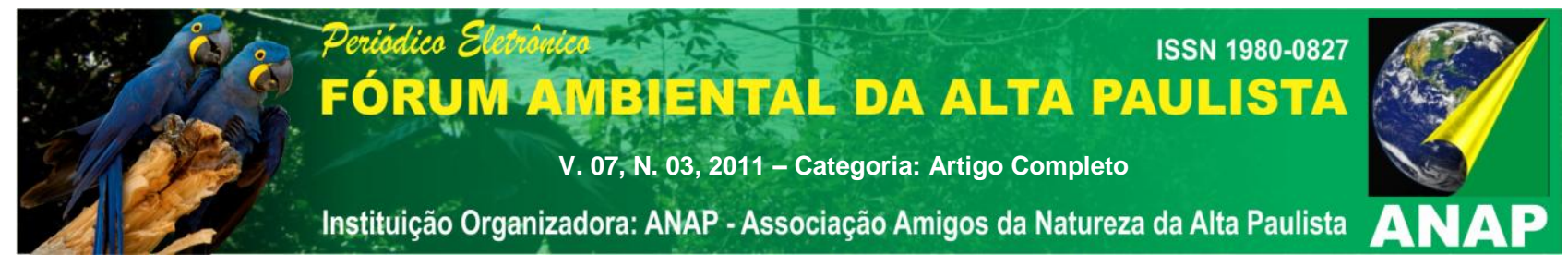

